\title{
Research and Application of predictive control based on state feedback theory in power plant control system
}

\author{
R D Kokate \\ MGM'S, Jawaharlal, Nehru Engg. \\ College, N-6 CIDCO New \\ Aurangabad Maharashtra India
}

\author{
L.M.Waghmare \\ Shri Guru Govind Singh Institute of \\ Technology Nanded Maharashtra India
}

\begin{abstract}
This paper purposed a new generalized predictive control based on state feedback theory for the large inertia and large delay characteristics of the discrete reheat steam temperature plant. The idea is to compensate the large inertia and delay characteristics of the plant by the state feedback theory and the generalized plant by predictive control. The simulation results show that that the new control system has good robustness and transient performance. So it is an effective control strategy for large delay industry process.
\end{abstract}

\section{Categories and Subject Descriptors}

MATLAB, SIMULINK and Model predictive toolbox

\section{General Terms}

Algorithms, Performance, Design, predictive control. Transfer function, controller.

\section{Keywords}

Generalized predictive control (GPC), Pole placement, Observer, controller, state feedback.

\section{INTRODUCTION}

We know that general control strategy is adopted in large scale power plants control systems, while many utilities are going through downsizing of their work forces as a means to stay competitive, the operational requirement for increased safety, reliability, and efficiency of the already stressed power system is at an all time high. As the demand of power is increasing, power plants are getting more complex and expansive to run. These have brought a great challenge to the research Community to develop new technologies that will benefit the utility industry. Modern control theory [1] [2] developed from 1960,s has more superiorities than classical control theory in many control areas, and it extract more and more important effect in modern control systems. Since 1990, some researches have done a great deal of theory researching work [3] [4] in modern control theory of power plant control system and acquired some achievements in combining new theory with the practice. Several researchers [6] [7] applied state feedback theory to power plant steam temperature control system and got the satisfying effect. So the control strategy based on state feedback [3] is an effective control method to the large inertia process. Predictive control is another effective control method for large delay process, which was put forward in the late 1970's. The application and research of predictive control had a good many developments [8] [10] in 1980's. The algorithm of predictive control [9] mostly includes DMC (Dynamic matrix control), MAC, GPC etc.

The state variable control and predictive can resolve the control problem of large delay process, combining the two advanced control strategy together could represent well. Aiming the practical problem of power plant reheat steam temperature control, we propose a new predictive control based on state feedback theory, which combined state feedback theory with predictive theory, and got fine effect of the local control and simulation. It proved that the predictive control based on state feedback theory is fit for boiler steam temperature control system, and it is effective strategy to large delay process control.

\section{GENRALISED PREDICTIVE CONTROL AND STATE FEEDBACK THEORY}

We have combined the state feedback and predictive control, for the unit reheat steam temperature's control. We deduce state variable and state feedback theory into predictive control algorithm putting forward the new predictive control based on state feedback theory.

In fig. 1 by selecting proper state feedback gain matrix $(k)$ it is possible system to have closed loop poles at the desired locations [5] [6] provided that the system is controllable.

After state feedback compensating the generalized reheat steam temperature model will show rapid dynamic characteristics even though original model has large inertia. State feedback control needs the state variable system, but in practice some of the state variables cannot be measured accurately. So we should reconstruct real plant.by a state observer and acquire the parameters of the plant and estimates the state variable based on the measurement of the output and control variables. 


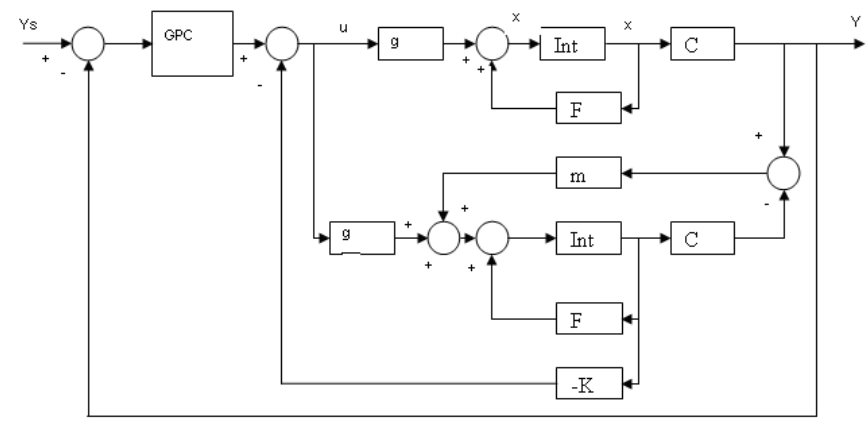

Fig.1. predictive control based on state feedback theory

Thus the whole design process of steam temperature Control systems has five steps.

1. Calculating the state feedback matrix $k$

2. Designing of full state observer and prediction observer

3. Computing observer gain matrix $m$

4. Designing observer-state feedback controller

5. Calculating parameters of GPC controllers for compensated generalized plant.

\subsection{DESIGN OF STATE FEEDBACK CONTROLLER}

The following steps are carried out to design state feedback controller.

\subsubsection{Calculating the State Feedback Matrix K}

Consider the discrete model of the plant.

$$
\begin{aligned}
x(k+1) & =f x(k)+g u(k) \\
y(k) & =c x(k)
\end{aligned}
$$

Where,

$x=$ state vector $=$ scalar input $=$ scalar output $=n x n$ real constant matrix $=n x l$ real constant matrix $=0,1,2 \ldots \ldots$

With state feedback control law, the resulting closed loop system (1) and (2) is given by (3) and (4),

$$
\begin{gathered}
u(k)=-k x(k) \\
k=\left[k_{1}, k_{2}, k_{3}---k_{n}\right] \\
x(k+1)=(f-g k(x)
\end{gathered}
$$

A necessary and sufficient condition for arbitrary placement of closed loop system is that the system (1) is completely controllable.

\subsubsection{Design of Full State Observer}

The purpose of this section is to show how to determine algorithms which will reconstruct all the states, given measurement of a portion of them, If the state is $x$ then the estimate $\hat{x}$ and the idea is to let $u=-k \hat{x}$ replacing true states by observer is shown in fig. 2

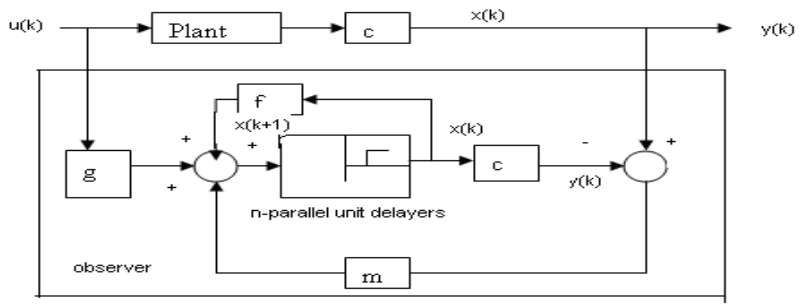

Fig.2 Block diagram Prediction observer

\subsubsection{Prediction Observer}

An estimation scheme employing a full order observer is shown in fig. 2 and the estimated equation is given by,

Let $u(k)=-k \hat{x}(\mathrm{k})$

$\hat{x}(k+1)=(f-m c) \hat{x}(k)+g u(k)+m y(k)$

Where $m$ is an $n x l$ real constant gain matrix. We will call this a prediction observer because the estimate $\hat{x}(k+1)$ is one sampling period ahead of the measurement $y(k)$.

A difference equation describing the behavior of the error is obtained by subtracting (5) from (1)

$$
x(k+1)-\hat{x}(k+1)=(f-m c)[x(k)-\hat{x}(k)]
$$

Let

$$
\begin{aligned}
& e(k+1)=x(k)-\hat{x}(k) \\
& e(k+1)=(f-m c) e(k)
\end{aligned}
$$

From (12) we see that the dynamic behavior of the error signal is determined by the eigenvalues of $(f-m c)$.If matrix $(f-m c)$ is stable matrix the error vector converges to zero for any initial error $e(0)$.

\subsubsection{Design of Prediction Observer}

The following design steps are followed for designing of full state prediction observer

Step1. Check the given system is completely state controllable and observable using

$$
N=\left[c^{T}: f^{T} c^{T}: \ldots \ldots . .\left(f^{T}\right)^{n-1} c^{T}\right]
$$

We also assumed that control law used is

$$
u(k)=-k \hat{x}(\mathrm{k})
$$

Where $\hat{x}(\mathrm{k})$ is observed state and $k=r x n$ matrix. The state observer dynamics are given by ,

$$
\begin{gathered}
\hat{x}(k+1)=f \hat{x}(k)+g u(k)+m[y(k)-\hat{y}(k)] \\
\hat{x}(k+1)=(f-m c) \hat{x}(k)+g u(k)+m c x(k)
\end{gathered}
$$

If the given system is controllable and observable and it is in canonical and observable form use transformation matrix equal to identity matrix (I) else transform the given system into observable canonical form using

Define

$$
o=\left(W N^{T}\right)^{-1}
$$

Where $w$ is 


$$
W=\left[\begin{array}{llllc}
\mathrm{a}_{n-1} & \mathrm{a}_{n-2} & \ldots & \mathrm{a}_{1} & 1 \\
\mathrm{a}_{n-2} & \mathrm{a}_{n-3} & \ldots & 1 & 0 \\
: & : & \ldots & : & \\
\mathrm{a}_{1} & 1 & \ldots & 0 & 0 \\
1 & 0 & \ldots & 0 & 0
\end{array}\right]
$$

Where $a_{1}, a_{2} \ldots \ldots \ldots a_{n}$ are the coefficient of the characteristics equation given by

$$
|z I-f|=0
$$

Step2. Let us define

$$
x(k)=Q \beta(k)
$$

Where $\beta(k)$ is an n-vector using (12) the (1) and (2) can be modified to

$$
\begin{aligned}
& \beta(k+1)=Q^{-1} f Q \beta(k)+Q^{-1} g u(k) \\
& y(k)=c Q \beta(k)
\end{aligned}
$$

where

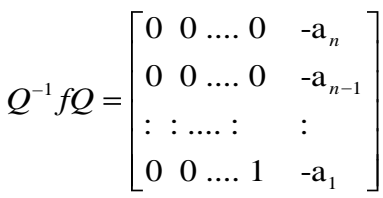

$$
\begin{aligned}
& c Q=\left[\begin{array}{lllll}
0 & 0 & \ldots . & 0 & 1
\end{array}\right]
\end{aligned}
$$

Now, define

$$
\hat{x}(k)=Q \beta(k)
$$

Then substituting (17) into (9) we have

$$
\hat{\beta}(k+1)=Q^{-1}(f-m c) Q \hat{\beta}(k)+Q^{-1} g u(k)+Q^{-1} m c Q \beta(k)
$$

Subtracting (19) from (17)

$$
\begin{aligned}
\beta(k+1)-\hat{\beta}(k+1)= & \left(Q^{-1} f Q-Q^{-1} m c Q\right)[\beta(k)-\hat{\beta}(k)] \\
& \text { Define } \\
& e(k)=\beta(k)-\hat{\beta}(k)
\end{aligned}
$$

Then equation (20) becomes.

$$
e(k+1)=Q^{-1}(f-m c) Q e(k)
$$

We require the error dynamics to be stable and $e(k)$ to reach zero with sufficient speed. If we require $e(k)$ to reach zero as fast as possible then we require then error response to be deadbeat. So we must select the eigen values of $(f-g c)$ to zero.

Notice that

$$
Q^{-1} m=\left[\begin{array}{cccccc}
\mathrm{a}_{n-1} & \mathrm{a}_{n-2} & \ldots & \mathrm{a}_{1} & 1 \\
\mathrm{a}_{n-2} & \mathrm{a}_{n-3} & \ldots . & 1 & 0 \\
: & : & \ldots & : & : \\
\mathrm{a}_{1} & 1 & \ldots & 0 & 0 \\
1 & 0 & \ldots & 0 & 0
\end{array}\right]\left[\begin{array}{l}
c \\
c f \\
: \\
c f^{n-2} \\
c f^{n-1}
\end{array}\right]\left[\begin{array}{l}
k_{1} \\
k_{2} \\
\vdots \\
k_{n-1} \\
k_{n}
\end{array}\right]
$$

where

$$
m=\left[\begin{array}{l}
k_{1} \\
k_{2} \\
: \\
k_{n-1} \\
k_{n}
\end{array}\right]
$$

Since $Q^{-1} m$ is n-vector then let us write

$$
Q^{-1} m c Q=\left[\begin{array}{l}
\delta_{n} \\
\delta_{n-1} \\
: \\
\delta_{1}
\end{array}\right]
$$

Then referring to (19) we have

$$
Q^{-1} m c Q=\left[\begin{array}{l}
\delta_{n} \\
\delta_{n-1} \\
: \\
\delta_{1}
\end{array}\right] 0 \quad 0 \ldots \quad 1=\left[\begin{array}{ccccc}
0 & 0 & \ldots . & 0 & \delta_{n} \\
0 & 0 & \ldots . & 0 & \delta_{n-1} \\
: & : & \ldots & : & : \\
0 & 0 & \ldots & 0 & \delta_{n}
\end{array}\right]
$$

Step3. Calculate the coefficient of the characteristics equation is given by

$$
\left|z I-Q^{-1}(f-m c) Q\right|=0
$$

From this equation. We find out $a_{1}, a_{2} \ldots . . a_{n}$ and $\alpha_{1}, \alpha_{2} \ldots . . \alpha_{n}$.

Step4. Calculate the observer gain matrix $\boldsymbol{m}$ as

$$
m=\left(W N^{T}\right)^{-1}\left[\begin{array}{l}
\delta_{n}-a_{n} \\
\delta_{n-1}-a_{n-1} \\
: \\
\delta_{1}-a_{1}
\end{array}\right]
$$

Equation (23) specifies the necessary observer feedback gain matrix $m$. fig. 3 shows alternative representation of the observedstate feedback control system. Once we select the desired eigenvalues the observer can be designed in a way similar to the method used in the case of the pole placement problem. The desired characteristics equation should be chosen such that the observer responds at least four or five times faster than the closed loop system. 


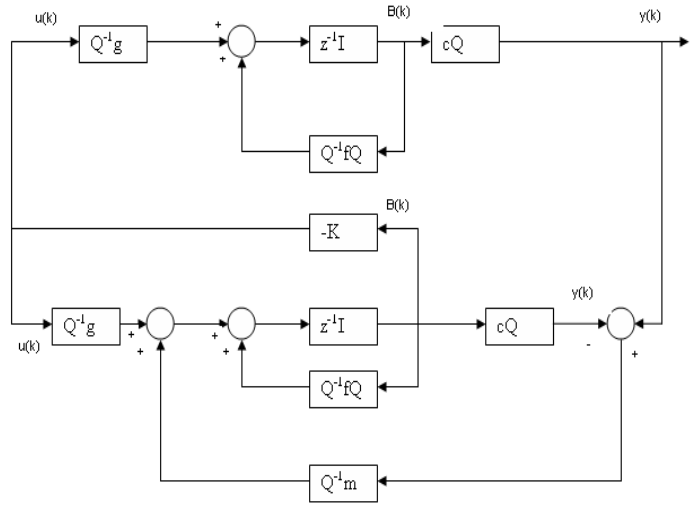

Fig.3 Alternative representation of observer-state feedback control system.

If we wish to leave deadbeat response the desired characteristics equation becomes

$z^{n}=0$

Comparing (22) and (24)

$$
\begin{aligned}
& a_{1}+\delta_{1}=\mathrm{O} \\
& a_{2}+\delta_{2}=\mathrm{O} \\
& : \\
& a_{n}+\delta_{n}=\mathrm{O}
\end{aligned}
$$

Hence for dead beat response

$$
m=\left[\begin{array}{l}
k_{1} \\
k_{2} \\
: \\
k_{n}
\end{array}\right]=Q\left[\begin{array}{l}
\delta_{n} \\
\delta_{n-1} \\
: \\
\delta_{1}
\end{array}\right]=\left(W N^{T}\right)\left[\begin{array}{l}
-a_{n} \\
-a_{n-1} \\
: \\
-a_{1}
\end{array}\right]
$$

\subsubsection{Designing of Observer state feedback Controller}

In this design the effect of the addition of the observer on closed loop system is considered. In pole placement design process, we assumed that the true state $x(k)$ was available for feedback. But in practice the true state $x(k)$ may not be measurable, so we will need to use the observed state $\hat{x}(k)$. Let us investigate the effects of the use of observed state $\hat{x}(k)$ rather than $\mathrm{x}(\mathrm{k})$ upon the characteristics equation of a closed loop control system.

Consider the completely state controllable and completely observable system defined by (1) and (2).For the state feedback control based on the observed state $\hat{x}(k)$ we have

$$
u(k)=-k \hat{x}(k)
$$

With this control the state equation becomes

$$
\begin{gathered}
x(k+1)=f x(k)-g K \hat{x}(k) \\
=(f-g K) x(k)+g K[x(k)-\hat{x}(k)]
\end{gathered}
$$

The difference between the actual state $\mathrm{x}(\mathrm{k})$ and observed state $\hat{x}(k)$ has been defined as the error $\mathrm{e}(\mathrm{k})$

$e(k)=x(k)-\hat{x}(k)$

Put the error vector e(k), (1) becomes

$$
\begin{aligned}
& x(k+1)=(f-g K) x(k)+g m(k) \\
& e(k+1)=(f-m c) e(k)
\end{aligned}
$$

Combining (27) and (28) we obtain

$$
\left[\begin{array}{c}
x(k+1) \\
e(k+1)
\end{array}\right]=\left[\begin{array}{ll}
\mathrm{f}-\mathrm{gk} & \mathrm{gk} \\
0 & \mathrm{f}-\mathrm{mc}
\end{array}\right]\left[\begin{array}{c}
x(k) \\
e(k)
\end{array}\right]
$$

This equation describing the dynamics of the observed state feedback control system.

The characteristics equation for the system is given by (30).

$|z I-f+g K||z I-f+m c|=0$

Notice that the closed loop poles of the observed state feedback control system consists of the poles due to the poles placement design plus poles due to observer design alone. This means that the pole placement and observer design are independent of each other. They can be designed separately and combined to form the observer state feedback control system.

\section{Formulation of Generalized Predictive Control (GPC)}

Before calculating the parameters of GPC first formulation is carried out [10], the different steps carried out in formulation are.

Most SISO plants when considering operation around particular set points and after linearization can be described by equation

$A\left(z^{-1}\right) y(t)=z^{-d} B\left(z^{-1}\right) u(t-1)+C\left(z^{-1}\right) e(t)$

Where $u(t)$ and $y(t)$ are the control and output sequence of the plant and $e(t)$ is a zero mean white noise $A, B$,and $C$ are the following polynomials in the backward shift operators.

$$
\begin{aligned}
& A\left(z^{-1}\right) y(t)=1+a_{1} z^{-1}+a_{2} z^{-1}+----+a_{n a} z^{-n a} \\
& B\left(z^{-1}\right) y(t)=b_{0}+b_{1} z^{-1}+b_{2} z^{-1}+----+b_{n a} z^{-n b} \\
& C\left(z^{-1}\right) y(t)=1+c_{1} z^{-1}+c_{2} z^{-1}+----+c_{n a} z^{-n c}
\end{aligned}
$$

Where $d$ is the dead time of the system. This model is known as CARIMA model. It has been argued that for many industrial applications in which disturbances are non-stationery an integrated CARIMA model is more appropriate.

$$
A\left(z^{-1}\right) y(t)=z^{-d} B\left(z^{-1}\right) u(t-1)+C\left(z^{-1}\right)+e(t) / \Delta
$$

With $\Delta=1-z^{-1}$

For simplicity $C$ polynomial in equation is chosen to be 1 .Notice that if $C^{-1}$ can truncated it can be absorbed into $A$ and $B$. 


\subsection{Minimization of cost function}

The Cost Function used in GPC algorithm consists of applying a control sequence that minimizes a multistage cost function of the form given by

$$
\mathrm{J}\left(\mathrm{N}_{1}, \mathrm{~N}_{2}, \mathrm{~N}_{\mathrm{u}}\right)=\sum_{\mathrm{j}=\mathrm{N}_{\mathrm{i}}}^{P} \partial(\mathrm{j})[\mathrm{Y}(\mathrm{t}+\mathrm{j} / \mathrm{t})-\mathrm{W}(\mathrm{t}+\mathrm{j})]^{2}+\sum_{\mathrm{j}=1}^{M} \lambda(\mathrm{j})[(\Delta \mathrm{u})(\mathrm{t}+\mathrm{j}-1)]^{2}(36)
$$

Where $y(t+j / t)$ is an optimum $j$-step ahead prediction of the system output on data up to time $k, N_{l}, P$ are the minimum and maximum costing horizons,$M$ control horizon $\delta(\mathrm{j})$ and $\lambda(\mathrm{j})$ are weighing sequences and $w(t+j)$ is the future reference trajectory which can be considered to be constant.The objective of predictive control is to compute the future control sequence $u(t), u(t+1),-----u\left(t+N_{u}\right)$ in such way that the future plant output $y(t+j)$ is driven close to $w(t+j)$ This is accomplished by minimizing $J\left(N_{l}, P, M\right)$. The Cost function minimization algorithm is given by,In order to minimize the cost function the optimal prediction of $y(t+j)$ for $j>N_{I}$ and $j>P$ is required to compute the predicted output consider the following Diophantine equitation.

$$
1=E_{j}\left(z^{-1}\right) A\left(z^{-1}\right)+z^{-j} F_{j}\left(z^{-1}\right) \text { with }---A\left(z^{-1}\right)=\Delta A\left(z^{-1}\right)
$$

The polynomials $E_{\mathrm{j}}$ and $F_{j}$ are uniquely defined with degrees $j-1$ and $n a$ respectively. They can be obtained dividing 1 by $A\left(z^{-1}\right)$ until the remainder can be factorized as $\mathrm{z}^{-\mathrm{j}} \mathrm{F}_{\mathrm{j}}\left(\mathrm{z}^{-\mathrm{j}}\right)$. An example demonstrating calculation $E_{\mathrm{j}}$ and $F_{j}$ coefficients in Diophantine equation is shown in example. If equation (2) is multiplied by $\Delta E_{j}\left(z^{-1}\right) z^{j}$

$$
A z^{-1} E_{j} z^{-1} y t+j=E_{j} z^{-1} B\left(z^{-1} \Delta u t+j-d-1+E_{j} z^{-1} \text { e } t+j\right.
$$

Considering (35) the (37) can be written as

$$
1-\mathrm{z}^{-\mathrm{j}} \mathrm{F}_{\mathrm{j}} \mathrm{z}^{-1} \text { y } \mathrm{t}+\mathrm{j}=\mathrm{E}_{\mathrm{j}} \mathrm{z}^{-1} B \mathrm{z}^{-1} \Delta \mathrm{u} \mathrm{t}+\mathrm{j}-\mathrm{d}-1+\mathrm{F}_{\mathrm{j}} \mathrm{z}^{-1} \text { y } \mathrm{t}+\mathrm{E}_{\mathrm{j}} \mathrm{z}^{-1} \text { e } \mathrm{t}+\mathrm{j}
$$

This can be written as

$$
y t+j=F_{j} z^{-1} y t+E_{j} z^{-1} B z^{-1} \Delta u t+j-d-1+F_{j} z^{-1} y t+E_{j} z^{-1} \text { e } t+j
$$

As the degree of polynomial $E_{j}\left(z^{-1}\right)=j-1$ the noise term in equation are used in all future. The best prediction of $y(t+j)$ is given equation by

$$
\mathrm{y}^{\mathrm{t}}+\mathrm{j}=\mathrm{G}_{\mathrm{j}} \mathrm{z}^{-1} \Delta \mathrm{u} \mathrm{t}+\mathrm{j}-\mathrm{d}-1+\mathrm{F}_{\mathrm{j}} \mathrm{z}^{-1} \text { y } \mathrm{t}
$$

Where

$$
\mathrm{G}_{\mathrm{j}} \mathrm{z}^{-1}=\mathrm{E}_{\mathrm{j}} \mathrm{z}^{-1} \mathrm{~B} \mathrm{z}^{-1}
$$

It is very simple to show that the polynomials $E_{j+1}$ and $F_{j}$ can be obtained recursively. Consider the polynomials $E_{j}$ and $F_{j}$ have been obtained by dividing as $z^{-j} F_{j}\left(z^{-1}\right)$. These polynomials can be expressed as

$$
\begin{gathered}
\mathrm{F}_{\mathrm{j}} \mathrm{z}^{-1}=\mathrm{f}_{\mathrm{j}, 0}+\mathrm{f}_{\mathrm{j}, 1} \mathrm{z}^{-1}+\mathrm{f}_{\mathrm{j}, 2} \mathrm{z}^{-2}+------\mathrm{f}_{\mathrm{j}, \mathrm{na}} \mathrm{z}^{-\mathrm{na}} \\
\mathrm{E}_{\mathrm{j}} \mathrm{z}^{-1}=\mathrm{e}_{\mathrm{j}, 0}+\mathrm{e}_{\mathrm{j}, 1} \mathrm{z}^{-1}+\mathrm{ef}_{\mathrm{j}, 2} \mathrm{z}^{-2}+------\mathrm{e}_{\mathrm{j}, \mathrm{na}} \mathrm{z}^{-(\mathrm{j}-n a)}
\end{gathered}
$$

Suppose that same procedure is used to obtain $\mathrm{E}_{\mathrm{j}+1}$ and $\mathrm{F}_{\mathrm{j}+1}$, that is dividing $l$ by $A\left(z^{-1}\right)$ until the remainder of the division can be factorized as $z^{-(j-1)} F_{j+1}\left(z^{-1}\right)$ with

$$
F_{j+1} z^{-1}=f_{j+1,0}+f_{j+1,1} z^{-1}+f_{j+1,2} z^{-2}+-------f_{j+1, n a} z^{-n a}
$$

It is clear that only another step of the division of the performed to obtain the polynomials $E_{j+1}$ and $F_{j+1}$ the polynomials $E_{j+1}$ will be given by

$$
E_{j+1} z^{-1}=E_{j} z^{-1}+e_{j+1} z^{-1}
$$

With $e_{j+1, j}=f_{j, 0}$. The coefficients of polynomials $f_{j+l}$ can then be expressed as:

$\mathrm{f}_{\mathrm{j}+1, \mathrm{i}}=\mathrm{f}_{\mathrm{j}+1, \mathrm{i}+1}-\mathrm{f}_{\mathrm{j}, 0} \mathrm{a}_{\mathrm{i}+1} \quad \mathrm{i}=0---\mathrm{na}-1$

The polynomials $G_{j+1}$ can be obtained recursively as follows:

$$
\begin{aligned}
& G_{j+1}=E_{j+1} B=E_{j}+f_{j, 0} z^{-1} B \\
& G_{j+1}=G_{j}+f_{j, 0} z^{-1} B
\end{aligned}
$$

That is the first $\mathrm{j}$ coefficient of $G_{j+l}$ will be identical to those of $G_{\mathrm{j}}$ and the remaining coefficients will be given by:

$$
g_{j+1, j+1=} g_{j, j+i}+f_{j, 0} b_{i} \text { for } i=0---n b
$$

To solve the GPC problem the set of control signals $u(t), u(t+1),---$ $---u(t+N)$ has to be obtained in order to optimize expression. As the system considered has a dead time $d$ sampling period, the output of the system will be influenced by signal $u(t)$ after sampling period $d+1$. The values $N_{l}, P, M$ defining the horizons can be defined by $P=d+N, P=d+N$ and $M=N$. Notice that there is no point in making $N_{l}>d+1$ as added to expression will only depend on the past control signals. on the other hand if $N_{l}>d+1$ the first point in the reference sequence, being the ones guessed with most certainly, will not be taken into account. Now consider the following set of $\mathrm{j}$ ahead optimal predictions given in equitation.

$$
\begin{aligned}
& \mathrm{Y} t+\mathrm{d}+1 / \mathrm{t}=\mathrm{G}_{\mathrm{d}+1} \Delta \mathrm{u} \mathrm{t}+\mathrm{f}_{\mathrm{d}+1} \mathrm{yt} \\
& \mathrm{Y} \mathrm{t}+\mathrm{d}+2 / \mathrm{t}=\mathrm{G}_{\mathrm{d}+2} \Delta \mathrm{u}+\mathrm{f}_{\mathrm{d}+2} \mathrm{yt} \\
& \cdot \\
& \mathrm{Yt}+\mathrm{d}+\mathrm{N} / \mathrm{t}=\mathrm{G}_{\mathrm{d}+\mathrm{N}} \Delta \mathrm{u} \mathrm{t}+\mathrm{N}-1+\mathrm{f}_{\mathrm{d}+\mathrm{N}} \mathrm{y}(\mathrm{t})
\end{aligned}
$$

This can be written as:

$$
\mathrm{Y}=\mathrm{Gu}+\mathrm{f} \mathrm{z}^{-1} \text { y } \mathrm{t}+\mathrm{G} \mathrm{z}^{-1} \Delta \mathrm{u} \mathrm{t}-1
$$

Where

$$
\begin{gathered}
y=\left[\begin{array}{l}
y(t+d+1) \\
y(t+d+2) \\
----- \\
y(t+d+N / t)
\end{array}\right], u=\left[\begin{array}{l}
\Delta u(t) \\
\Delta u(t+1) \\
----- \\
\Delta u(t+N-1)
\end{array}\right], G=\left[\begin{array}{l}
g_{0}-0----0 \\
g_{1}-g_{0}----0 \\
-------- \\
g_{N-1}-g_{N-2}--g_{o}
\end{array}\right] \\
G(z)=\left[\begin{array}{l}
\left(G_{d+1}\left(z^{-1}\right)-g_{0}\right) z \\
\left(G_{d+1}\left(z^{-1}\right)-g_{0}-g_{1} z^{-1}\right) z^{2} \\
---- \\
\left.\left(G_{d+N}\left(z^{-1}\right)-g_{0}-g_{1} z^{-1}---g_{N-1} z^{-(N-1)}\right) z^{N}\right)
\end{array}\right], F\left(z^{-1}\right)=\left[\begin{array}{l}
F_{d+1}\left(z^{-1}\right) \\
F_{d+2}\left(z^{-1}\right) \\
--- \\
F_{d+N}\left(z^{-1}\right)
\end{array}\right]
\end{gathered}
$$

Note that if the plant dead time is $d>1$ the first $d-1$ rows of $G$ will be null, but if instead $N_{l}$ is assumed to be equal to d the 
leading element is non-zero.Howerer, as $d$ will not in general known in the self tuning case one key feature of the GPC approaches is that a stable solution is possible even if the leading rows of $G$ are zero.

Notice that the last two terms in (16) only dependent on the past and can be grouped into f leading to:

$\mathrm{Y}=\mathrm{Gu}+\mathrm{f}$

Notice that if all initial conditions are zeros the free response $\mathrm{f}$ is also zero. If a unit step is applied to the input at time t i.e. $(t)=1$, $\Delta u(t+1)=0, \ldots \quad \Delta u(t+N-1)=0 \quad$ he expected output sequence $[y(t+1), y(t+2) \ldots y(t+N)]^{T}$ is equal to the first column of matrix $G$.That is the first column of matrix $\mathrm{G}$ can be calculated as the step response of the plant is applied to the manipulated variable. The free response term can be calculated by (18).

$$
\mathrm{F}_{\mathrm{j}+1}=\mathrm{z}\left(1-\mathrm{A} \quad \mathrm{z}^{-1} \quad \mathrm{f}_{\mathrm{j}}+\mathrm{B} \mathrm{z}^{-1} \quad \Delta \mathrm{u} \mathrm{t}-\mathrm{d}+\mathrm{j}\right.
$$

With $f_{0}=y(t)$ and $\Delta u(t+j)=0$ for $j>0$

Expression (5) can be written as

$$
\mathbf{J}=\mathrm{Gu}+\mathrm{f}-\mathrm{w}^{\mathrm{T}} \mathrm{Gu}+\mathrm{f}-\mathrm{w}+\lambda \mathbf{u}^{\mathrm{T}} \mathbf{u}
$$

Where

$$
\mathrm{W}=\left[\begin{array}{llllll}
\mathrm{w} & \mathrm{t}+\mathrm{d}+1 & \mathrm{w} & \mathrm{t}+\mathrm{d}+2 & \ldots \ldots \mathrm{w} & \mathrm{t}+\mathrm{d}+\mathrm{N}
\end{array}\right]^{\mathrm{T}}
$$

Where it has been considered that the future reference trajectory keeps constants along the horizon or its evolution is unknown and therefore $w(t+i)=w(t)$.Equitation (52)can be written as

$$
\begin{aligned}
\mathbf{J} & =\mathbf{1} / 2 \mathbf{u}^{\mathrm{T}} \mathbf{H u}+\mathbf{b}^{\mathrm{T}} \mathbf{u}+\mathbf{f}_{\mathrm{o}} \\
\mathrm{H} & =2 \mathrm{G}^{\mathrm{T}} \mathrm{G}+\mathrm{RI} \\
\mathrm{b}^{\mathrm{T}} & =2 \mathrm{f}-\mathrm{w}^{\mathrm{T}} \mathrm{G} \\
\mathrm{f}_{\mathrm{o}} & =\mathrm{f}-\mathrm{w}^{\mathrm{T}} \mathrm{f}-\mathrm{w}
\end{aligned}
$$

The minimum of $\mathbf{J}$ assuming there are no constraints on the control signals can be found by making the gradient of $\mathbf{J}$ equal to zero which leads to

$$
\begin{aligned}
& \mathbf{u}=-\mathbf{H}^{-1} \mathbf{b} \\
&=\mathbf{G}^{\mathrm{T}} \mathbf{G}+\lambda \mathbf{I}^{-1} \mathbf{G}^{\mathrm{T}}(\mathbf{w}-\mathbf{f})
\end{aligned}
$$

The dimensions in the matrix involved in equitation (20) is $N * N$ .Although in the nonadaptive case the inversion need be performed only once. In a self tuning version the computational load of inverting at each sample would be excessive .Moreover if the wrong value for the dead time is assumed $G^{T} G$ is singular and hence a finite nonzero value of weighing $\lambda$ would be required foe a realizable control law which is inconvenient because the accurate value for $\lambda$ would not be known a piror notice that the control signal that is actually sent to the process is the first element of vector $u$ which is given by (55).

$$
\Delta \mathrm{u}=\mathrm{k} \quad \mathrm{w}-\mathrm{f}
$$

Where $k$ is the first row of matrix $\left(G^{T} G+\lambda I\right)^{-1} G^{T}$.If there are no future predicted errors that is $(w-f)=0$, then there is no control move. Since the objective will be fulfilled with the evaluation of the process. However in other case there will be an increment in the control action proportional (with factor $\mathrm{k}$ ) to the future errors. Notice that the action is taken with respect to the future errors as is the case in conventional feedback controllers.

Notice that only the first element of $u$ is applied then at next sampling instant new data are acquired and a new set of control move is calculated once again only the first control move is implemented. These activities repeated at each sampling instant and the strategy is receding horizon approach. It may strange to calculate an $M$ step control policy and then only implement the first move. The important advantage of receding horizon approach is that new information in the form of the most recent measurements $y(k)$ is utilized immediately instead of being ignored for the next $M$ sampling intants.otherwise the multistep predictions and control moves would be used on old information and thus be adversely affected by unmeasured disturbances.

\section{MODIFIED GPC-OBSERVER CONTROLLER}

Fig shows the modified GPC-Observer controller which is connected in cascaded to obtain combined effect of both controllers and the time response obtained from the above controller gives excellent results as compared to individual controllers used for control of plant shown in Fig.8.

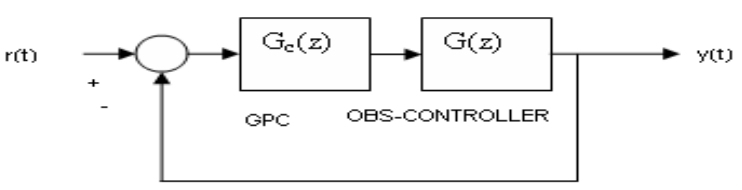

\section{Fig. 8 Block diagram GPC-Observer state feedback controller.}

\section{SIMULATION RESULTS}

The Reheating temperature control model is considered from [3] and simulation is carried out using GPC Control Based on State Feedback Theory.

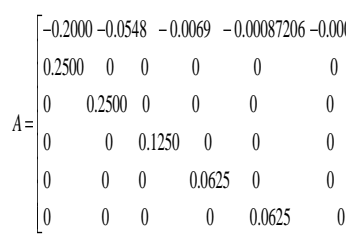

$$
\begin{aligned}
& B=\left[\begin{array}{l}
0.0020 \\
0 \\
0 \\
0 \\
0 \\
0
\end{array}\right] \\
& \mathrm{C}=\begin{array}{lllll}
0 & 0 & 0 & 0 & 0-0.00093056
\end{array}
\end{aligned}
$$

The above model converted in to discrete model with sampling time of $6.5 \mathrm{sec}$, the transfer function of discrete plant is given by, 
$G_{d}(z)=\frac{-4.837 \mathrm{e}-009 z^{\wedge} 5-2.304 \mathrm{e}-007 z^{\wedge} 4-1.018 \mathrm{e}-006 z^{\wedge} 3-8.456 \mathrm{e}-007 z^{\wedge} 2-1.32 \mathrm{e}-007 z-1.912 \mathrm{e}-009}{z^{\wedge} 6-4.923 z^{\wedge} 5+10.04 z^{\wedge}-10.84 z^{\wedge} 3+6.528 z^{\wedge} 2-2.078 z+0.2725}$

The discrete plant compensated by state feedback $(k)$ for desired eigen values is given by,

$G_{c}(z)=\frac{-4.837 \mathrm{e}-009 z^{\wedge}-2.304 \mathrm{e}-007 z^{\wedge}-1.018 \mathrm{e}-006 z^{\wedge} 3-8.456 \mathrm{e}-007 z^{\wedge}-1.32 \mathrm{e}-007 z-1.912 \mathrm{e}-009}{z^{\wedge}+0.2591 z^{\wedge} 5+0.02894 z^{\wedge} 4+0.001902 z^{\wedge} 3+6.614 \mathrm{e}-005 z^{\wedge} 2+5.723 \mathrm{e}-007 z+1.885 \mathrm{e}-009}$

The response to initial conditions of $x(0)$ and $e(0)$ is shown in fig. 4 for the desired eigen values selected for discrete plant equation(57).

$$
\begin{aligned}
& \mu_{1,2}=-0.0053022 \pm j * 0.003341, \\
& \mu_{3,4}=-0.033736 \pm j * 0.068634 \\
& \mu_{5}=-0.087338 \\
& \mu_{6}=-0.092728
\end{aligned}
$$

The response to initial condition seems to be acceptable. It means the selected eigen values (poles) are acceptable. It also confirms the designed observer state feedback controller is stable.
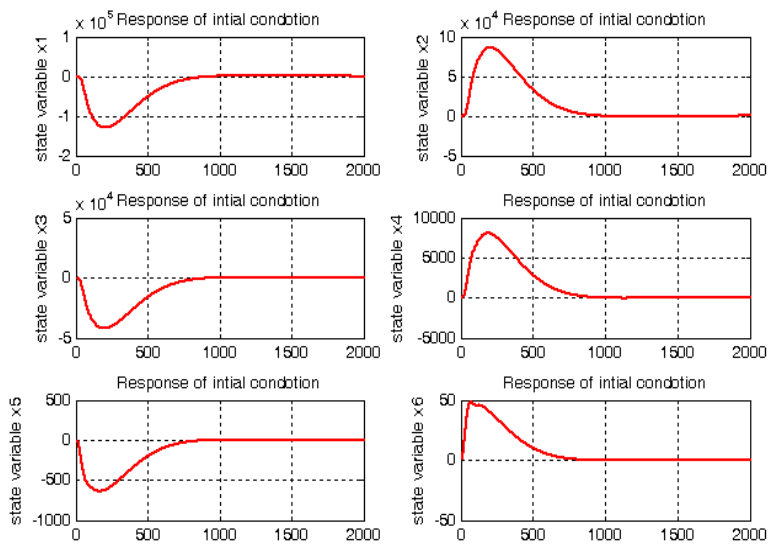

Fig.4 Response to initial condition of discrete plant.

The actual states and estimated states of reheat Temperature control system of discrete plant shown in fig.5
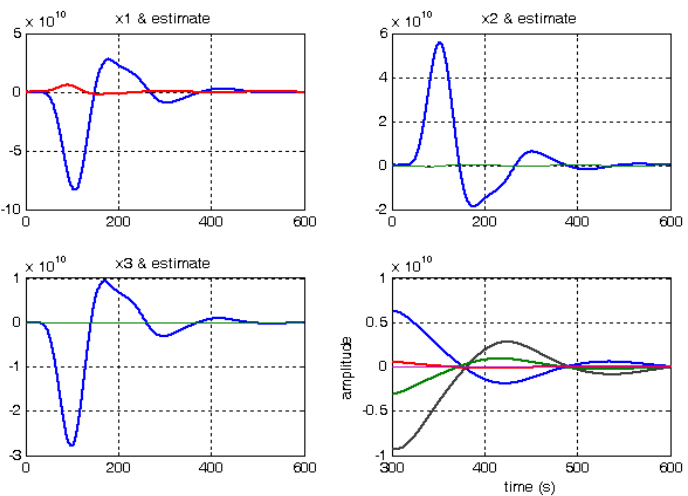

Fig.5 Response of actual observer states and estimated states of reheat steam temperature Control of discrete plant.

For the above desired eigen values the state feedback gain matrix is given by,

$k=\left[\begin{array}{llllll}183.6094 & 337.36733 & 327.9824 & 422.4545 & 686.2259 & 537.7020\end{array}\right]$
After working with state feedback gain matrix $K$ we have find the step disturbance on reheat steam temperature model before compensation after compensation shown figure. 6 and figure. 7.

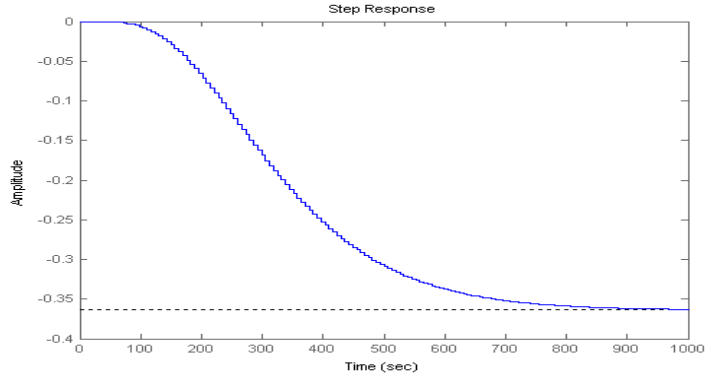

Fig.6 the step response curves for generalized reheat steam temperature plant before compensation.

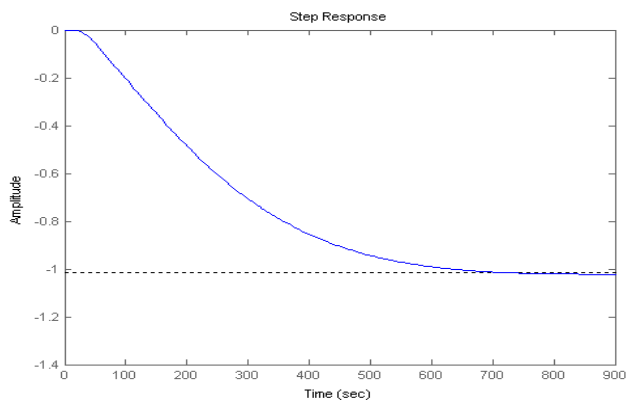

Fig.7 the step response curves for generalized reheat steam temperature plant after compensation.

Compare fig.6 and fig.7 open loop steady time constant decreases $900 \mathrm{sec}$ to $700 \mathrm{sec}$.The response speed of reheat steam temperature plant has become rapid after compensation.

The observer gain matrix is obtained using desired eigen values given by (59)

$\mathrm{m}=1.0 \mathrm{e}+004 *\left[\begin{array}{c}0.1637 \\ -0.1077 \\ -2.5726 \\ -3.9876 \\ -1.7785 \\ -0.5569\end{array}\right]$

The transfer function of observer state feedback controller is obtained considering state feedback and observer gain matrix

$G(z)=\frac{-4.0540007 z^{\wedge} 5+1.3090008 z^{\wedge} 4-1.753 e 008 z^{\wedge} 3+1.19900088 z^{\wedge 2}-4.146 e 007 z+5.766 e 006}{z^{\wedge} 6+5.442 z^{\wedge} 5+16.68 z^{\wedge} 4+29.67 z^{\wedge} 3+19.11 z^{\wedge} 2+2.811 z+0.04045}$

fig.9 shows the step response of state feedback and observer controller which is not stable and fig. 10 shows response of Generalized predictive controller and observer controller for different set point also fig.11 shows the stability of modified controller as the poles of modified controller lies unit circle in $\mathrm{z}$ plane. 
The GPC design parameters selected are prediction horizon 80; control horizon 20 and weights on manipulated variable and output variable are Input rate 0.1 and output rate 1 .

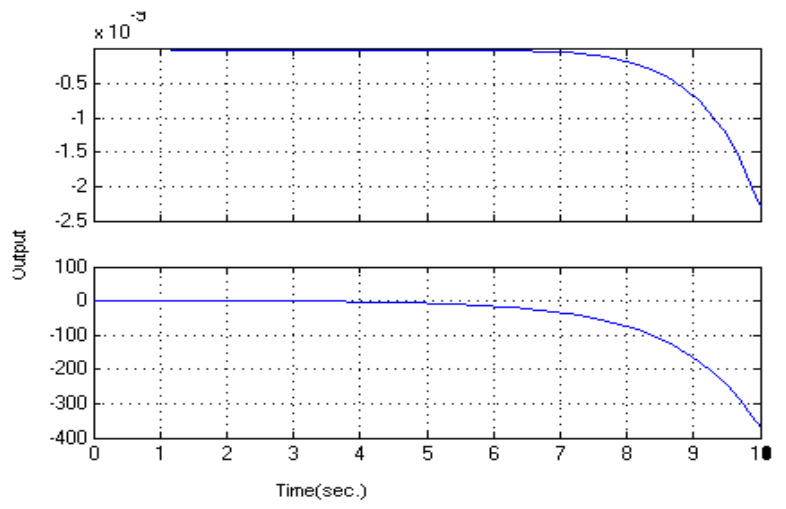

Fig.9 Response of State feedback and observer controller for step input.

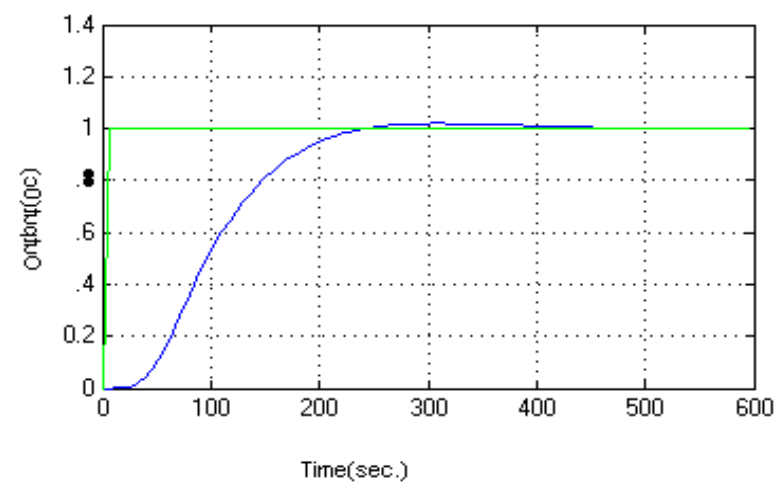

Fig.10 Response of GPC-State feedback controller for step input.

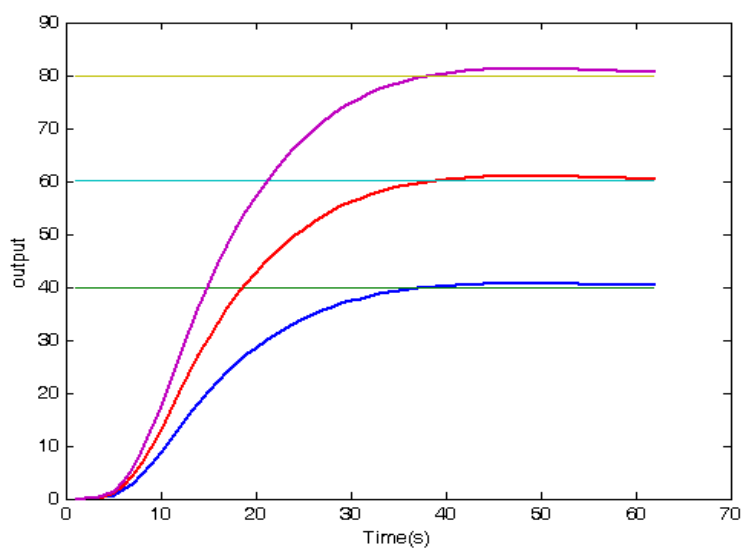

Fig.11 Response of GPC-State feedback controller for set point of 40, 60, $80 \dot{c}$

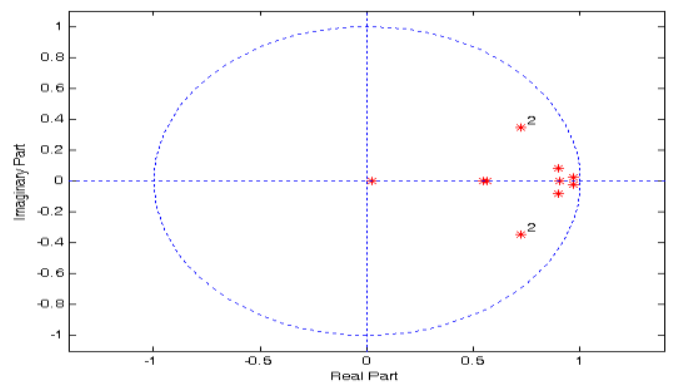

Fig.12 the response of closed

lo

Plant Output: MO1, Temp.

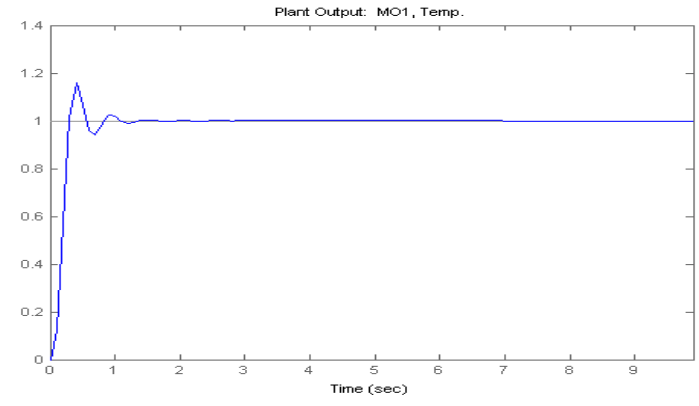

op poles of observer controller

Fig.13 Response of GPC-observer controller for step input

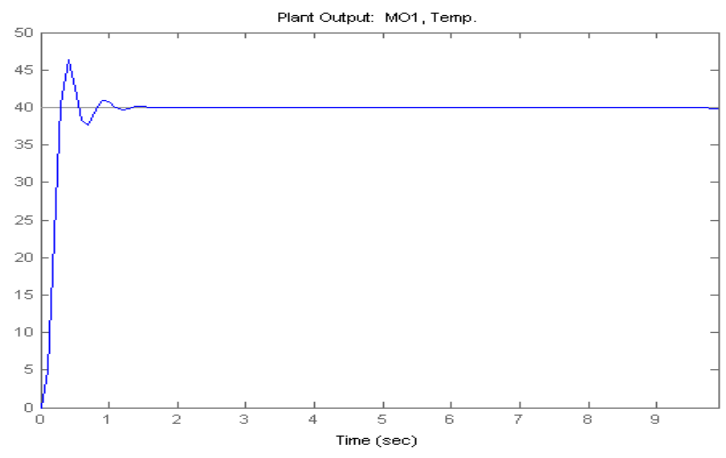

Fig.14 Response of GPC-observer controller for set point of $40 \dot{c}$

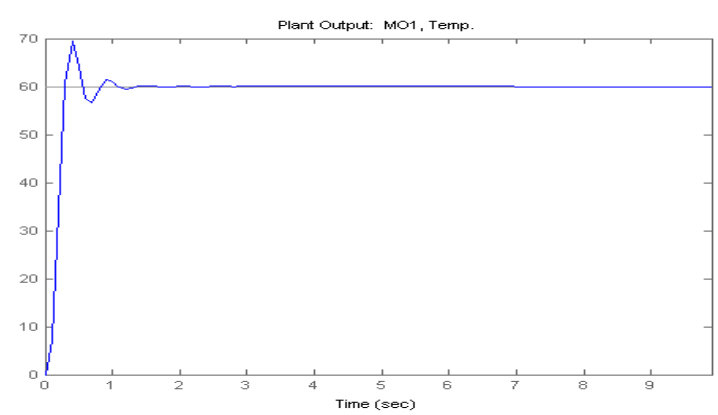

Fig.15 Response of GPC-observer controller for set point $60 \dot{\mathbf{c}}$ 


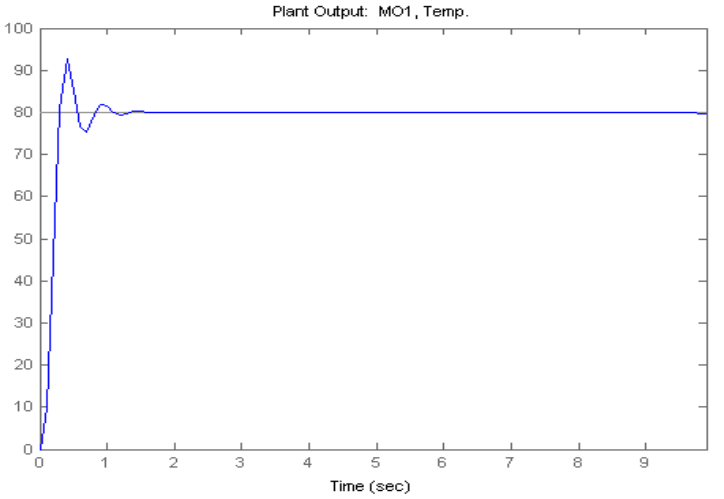

Fig.16 Response of GPC-observer controller for set point $80 \dot{c}$

\section{CONCLUSION}

We know that only the advanced system can improve the plant dynamic behavior to get efficient control of temperature of reheating plant. Predictive control theory based on state feedback theory is effective to control the thermal process with large inertia and large delay. It is observed in simulation study that the independent state feedback control is not sufficient to control the dynamics of plant but if we combined the predictive control and state feedback the simulation results are excellent in terms of settling time and stability of reheating temperature control system.

\section{REFERENCES}

1. Katsuhiko ogata, Discrete-Time control system, second edition Pearson India 2002.

2. Katsuhiko ogata, Modern control engineering, fourth edition prentice Hall of India New Delhi-110001 2005.
3. Hua Zhi-gang, HUA Hua. Reasearch and application of new predictive control based on state feedback theory in power plant application,IEEE Congress on Evolutionary computation(CEC-2007).

4. Han zhongxu Lv Xiuhong, Han Li Thee Robustness analysis of the state observer in the boiler super heater temperature control,[J].proceedings of the Chinese society for electrical Engineeering.23 (2003) 177-180.

5. Han znongxu. "An algebraic analysis method for state feedback pole configuration on second order System of higher order", [J]1 .Proceedings of the Chinese society for electrical Engineering. 22 (2002) 146-149.

6. Liu jain hung Yuan zhen. ,Reheat temperature control system Based on state variable controller, $[\mathrm{J}]$. Electric power.35 (2002)53-56

7. Han zhongxu. Application of state observer and state feedback in sub-critical boiler stem temperature control system,[J].proceedings of the Chinese for electrical 19 (1999) 76-79.

8. Tsang T.T.C, Clarke D.W. Generalized predictive control with constrains, proc.IEE 135 (1988) 451-460.

9. Brain Aufderheid,B.Wayne Bequette“.Extension of dynamic matrix control to multiple models," [J]. Computer and chemical Engineering 27 (2003) 10791096.

10. E.F. Camacho, C. Bordons, Model Predictive Control in the Process industry, Springer - Velag, London, 1995. 